\title{
Uteroplacental acute atherosis in preeclamptic pregnancies: Rates and clinical outcomes differ by tissue collection methods
}

\author{
Patji Alnaes-Katjavivi ${ }^{a, *}$, Borghild Roald ${ }^{\mathrm{b}}$, Anne Cathrine Staff ${ }^{\mathrm{a}}$ \\ ${ }^{a}$ Division of Obstetrics and Gynaecology, Oslo University Hospital, Ullevål and Faculty of Medicine, University of Oslo, Norway \\ ${ }^{\mathrm{b}}$ Department of Pathology, Oslo University Hospital, Ullevål and Faculty of Medicine, University of Oslo, Norway
}

\section{A R T I C L E I N F O}

\section{Keywords:}

Placenta

Decidua

Acute atherosis

Morphology

Immunohistochemistry

Preeclampsia

\begin{abstract}
A B S T R A C T
Objectives: Acute atherosis (AA) is a uteroplacental spiral artery lesion, identified by intramural lipid-laden foam cells, with highest rates in preeclampsia (PE). We compared AA detection rates in preeclampsia (PE) across three different decidual spiral artery collection methods in same patients. We tested whether the rate and topographical distribution of AA associates with clinical parameters.

Study design: Three decidual tissue types were harvested from each of 107 preeclamptic women delivered by cesarean section. Routine sampled basal surface placenta (decidua basalis, DB) and fetal membrane roll (decidua parietalis, DP) biopsies were compared with decidual vacuum suction biopsies (DB), regarding spiral artery rate and AA presence. Spiral arteries and AA were identified using predefined, immunohistochemically based criteria on serial sections.

Main outcome measures and results: Detection of spiral arteries (87\%) and AA (35\%) was highest in DB samples collected by vacuum suction compared to the two other methods. Pregnancies with AA detected in vacuum suctioned DB had lower gestational age at delivery, lower birth weight percentile and more often fetal growth restriction. Basal plate DB samples demonstrating AA associated with pregnancies affected by pathological fetal Dopplers, whereas AA detected in DP membrane rolls, did not.

Conclusions: Placental bed vacuum suction provides more spiral arteries and higher AA rate, suggesting underestimation of AA in conventional pathology samples of basal plate DB biopsies and DP. The association of AA with PE-related clinical parameters varies according to tissue collection method. Longitudinal studies could elucidate whether AA also identifies women with future premature cardiovascular risk.
\end{abstract}

\section{Introduction}

Acute atherosis (AA) is a histological lesion of uteroplacental arteries first characterized by Hertig in 1945 [1]. AA is focal and found in both myometrial [2] and decidual parts $[1,3]$ of the spiral arteries.

AA is most commonly associated with preeclamptic (PE) pregnancies [4], with rates varying between studies, from $10 \%$ to $38-41 \%$ [5-7]. This variation may be due to different tissue collection methods, heterogeneous patient groups, as well as lack of uniform and clear criteria for AA diagnosis. AA remains a lesion of unclear etiology and clinical significance, although its association to downstream placenta lesions is documented [3].

As well as being associated with pregnancy complications (e.g. placental insufficiency, placental abruptio, eclampsia), preeclampsia is also associated with health complications later in life, in particular morbidity from hypertension and atherosclerosis [8-10]. Acute atherosis bears some morphological similarities to early stage atherosclerosis [11], and our group has proposed that AA could represent an accelerated form of vasculopathy, developing during the short time of pregnancy at the fetal-maternal interface, and arising from local excessive inflammation as the final common pathway [12]. What remains to be clarified is whether the presence of decidual AA across various sampling sites differs in the association to pregnancy-related morbidity.

We have previously defined minimum inclusion criteria for a predefined and simple diagnosis of AA, allowing for reliable comparison of AA rates between different sample types, patient groups, and between different investigators [6]. Decidua is the name of the endometrium of pregnancy, and is normally found underneath the placenta (decidua

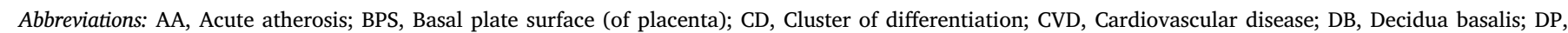

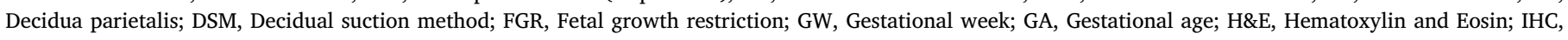
Immunohistochemistry; MR, (Fetal) membrane rolls; PE, Preeclampsia; PAS, Periodic acid Schiff

* Corresponding author at: Division of Obstetrics and Gynaecology, Oslo University Hospital, Ullevål, PO Box 4956 Nydalen, NO-0424 Oslo, Norway.

E-mail address: alkp@ous-hf.no (P. Alnaes-Katjavivi). 
basalis) or on the uterine wall outside the placenta (decidua parietalis). Our criteria for AA diagnosis were developed studying vacuum suction samples of decidua basalis tissue (DB) from the placental bed following the removal of the placenta during pre-labor cesarean section [7]. Previously, we have shown that our vacuum suction method provides a higher yield of decidua basalis spiral arteries (present in $86 \%$ of tissue sections evaluated), than in decidua basalis derived from placenta bed biopsies and maternal placental plate sections $(61 \%$ and $48 \%$, respectively [7]). However, no previous studies have compared these tissue collection methods from the same pregnancies [7].

Most preeclamptic women will deliver vaginally, making vacuum suction of decidua basalis not easily feasible. Consequently, conventional sampling of placenta and fetal membranes by the pathologist for decidual AA detection may be more feasible in the everyday clinical setting. No previous studies, including our own [7], rate AA detection in decidua sampled from the same person, comparing vacuum suction collection to conventional methods. The aim of this paper was therefore to compare the rates of spiral artery and AA presence in decidual collected by three different collecting methods in PE women. We hypothesized that the rate of AA detection differs depending on the collecting method of decidual tissue utilized, and that any association of AA detection with severity of $\mathrm{PE}$ and placental function will differ according to the decidual tissue type sampled.

\section{Methods}

\subsection{Patient recruitment, biopsy tissue sampling and preparation}

We included 107 preeclamptic women from the Oslo Pregnancy Biobank (2000-2016, www.oslo-universitetssykehus.no/opb) to the present study. Several of the women (88/107) had also participated in a previous decidua basalis (vacuum suction) acute atherosis study [6]. Preeclampsia (PE) was defined as new onset hypertension (blood pressure $\geq 140$ and/or $\geq 90 \mathrm{mmHg}$ ) and new onset proteinuria ( $\geq 1+$ on dipstick, or $\geq 30$ protein/creatinine ratio) $\geq 20$ weeks of pregnancy [13]. None of the women participating in the present study had any known chronic disease. Gestational week (GW) at delivery was defined by routine ultrasound screening at GW 17-20. Newborn gender specific baby weight percentiles were calculated according to Norwegian fetal growth curves [14]. Pathological fetal Dopplers were defined as abnormal PI in umbilical artery and/or middle cerebral artery ( $>2 \mathrm{SD}$ ), and/or cerebroplacental ratio $<1.08[15,16]$. All women provided informed written consent. The study was approved by the Regional Committee for Medical and Health Research Ethics.

Decidual tissue was sampled following delivery by pre-labor cesarean section [6], with intact membranes. Fig. 1. illustrates the three different sampling sites for decidual tissue. Decidua basalis (DB) tissue from the placental bed was collected both by the decidual vacuum suction method (DSM) developed by our group [7,17], and by routine basal plate placenta section (BPS) excised from macroscopically normal looking maternal plate placental tissue. From the same deliveries, decidua parietalis (DP) was collected through routinely prepared placental fetal membrane rolls (MR). The sections from both BPS and MR were prepared by the same pathology technician and according to the standardized routine for our University Hospital's Department of Pathology, as were the immunohistochemical stainings. All samples were fixated in $4 \%$ buffered formaldehyde and preserved in paraffin blocks from which slides were prepared as published previously [18].

\subsection{Decidual spiral artery identification by immunohistochemistry (IHC)}

For each pregnancy $(n=107)$, slides from each of the three tissue types were at first studied microscopically in H\&E stained sections for identification of spiral arteries, followed by the immunohistochemical verification of mural foam cells (acute atherosis). The stains and antibodies for IHC are listed in Supplementary Table 1 . Serial slides $(3 \mu \mathrm{m}$ thick) were prepared from each biopsy tissue-type, minimizing alteration in tissue topography between the first H\&E stained slides (used for initial identification of arteries and vacuolated arterial wall cells), and the subsequent immunostaining of slides with CD68 (used to confirm presence of lipid scavenger receptor, typically of macrophages and foam cells). Thus, at least two serial decidual slides per pregnancy were evaluated to confirm the presence or absence of spiral arteries and, thereafter, the presence or absence of acute atherosis, for each of the three tissue sampling methods.

Spiral arteries were detected first on H\&E-stained slides according to morphological features, assisted - if necessary - by identification of intramural smooth muscle (Desmin), and/or its replacement by fibrinoid change (intramural PAS-positive staining detected beyond the normal PAS staining of subendothelial basement, see Fig. 2) [19].

For decidua basalis samples (either collected by vacuum suction or from the placental basal plate sections), spiral arteries were considered representative if $\geq 140 \mu \mathrm{m}$ in diameter, avoiding inclusion of the smaller decidual nutrient ("straight") arteries (typically $<100 \mu \mathrm{m}$ in diameter) $[20,21]$. Inter- and intra-observer scoring for AA agreement between authors in decidua basalis tissue, according to our predefined criteria, has been shown previously to be substantial [6].

Spiral arteries belonging to the decidua parietalis lie beyond the area of endovascular trophoblast invasion and therefore are not expected to demonstrate features of trophoblast-assisted arterial remodeling [22]. Hence, for the biopsy tissues of placental fetal membrane rolls, smaller arteries of $<140 \mu \mathrm{m}$ in diameter were also included in our evaluation for AA in the decidua parietalis.

Acute atherosis was identified as previously [6], where $\geq 2$ adjacent vacuolated intramural cells visualized by H\&E were confirmed as foam cells by positive immunostaining with CD68 on serial slides (for the detection of the scavenger receptor expressed by foam cells and macrophages). Representative images for normal and AA-affected spiral arteries are demonstrated, by tissue-type category, in Fig. 2.

Rates of AA detection per tissue type sampled were based on samples where at least one artery section with AA was identified.

Data were analyzed using SPSS version 22 . The Chi squared test was used for dichotomous variables, and the Mann-Whitney-Wilcoxon test for continuous variables. Associations between AA presence per tissue category and clinical outcome were tested using Spearman correlation and bivariate logistic regression. A probability of $\leq 0.05$ was considered statistically significant.

\section{Results}

3.1. Comparison of detection of spiral arteries and acute atherosis in three decidua tissue types

Table 1 presents spiral artery and AA detection rates for the three uteroplacental tissue types. In five women no arteries were detectable in any of their three decidual tissue samples. Of the 107 preeclamptic women, spiral arteries were thus detected in 102 of the 107 preeclamptic women, with acute atherosis detected in 36\% (37 of 102), when all three tissue types sampled were combined for evaluation of AA.

Decidua basalis sections from vacuum tissue sampling detected the highest rate of spiral arteries (DSM: at least one spiral artery section detected in $87 \%$ of sections), followed by decidua parietalis tissue sections from fetal membranes (MR: 71\%). These were significantly higher than spiral artery detection rate in decidua basalis tissue from placental basal plate sections (BPS: $50 \%$ ), all p $<0.05$ (Table 1). In addition to identifying the highest rate of spiral arteries, vacuum collection samples of decidua basalis also identified the significantly highest rate of AA (Table 1: 35\%). Combining DSM with MR tissue increased both the rate of women where decidual spiral arteries are detected, and the rate of women with AA presence (Table 1).

There is overlap in AA detection between the three tissue collection 


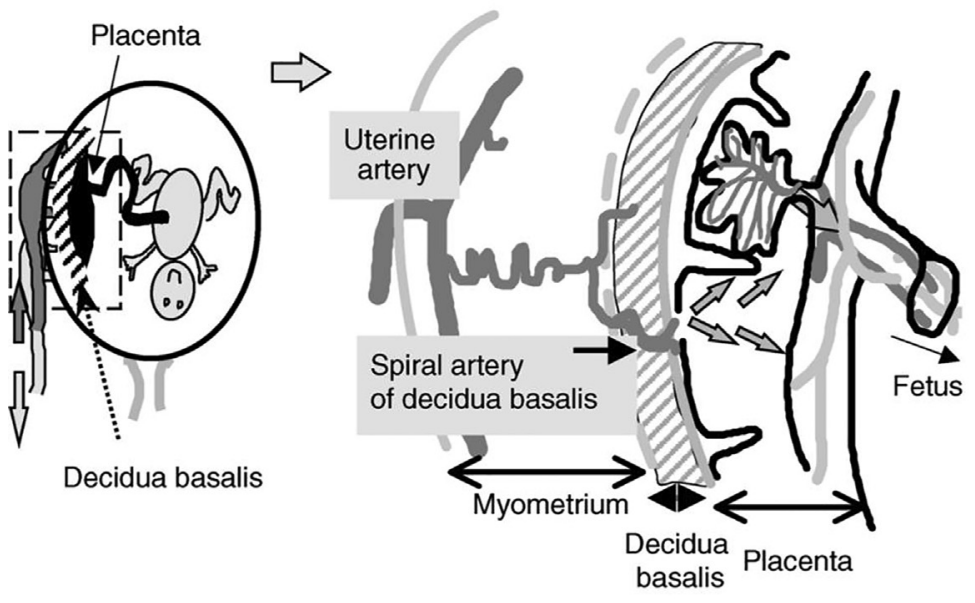

(a)

(b)

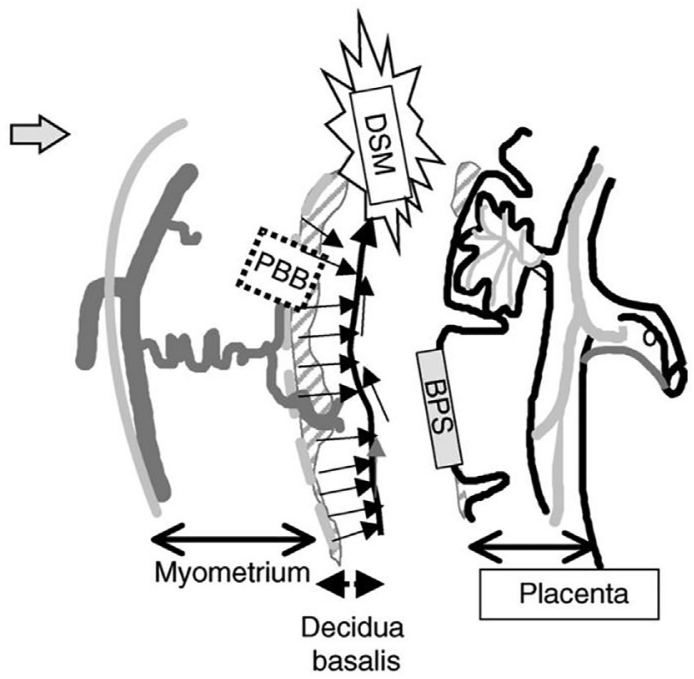

(c)

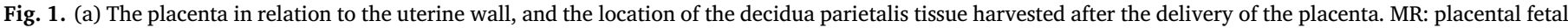

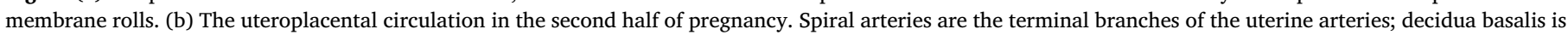



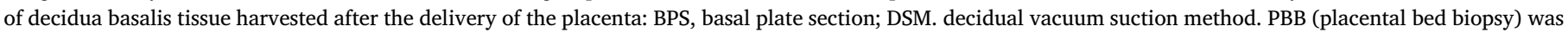
not tested in our present study. Revised from our previous Figure [7]; with permission from the Journal).

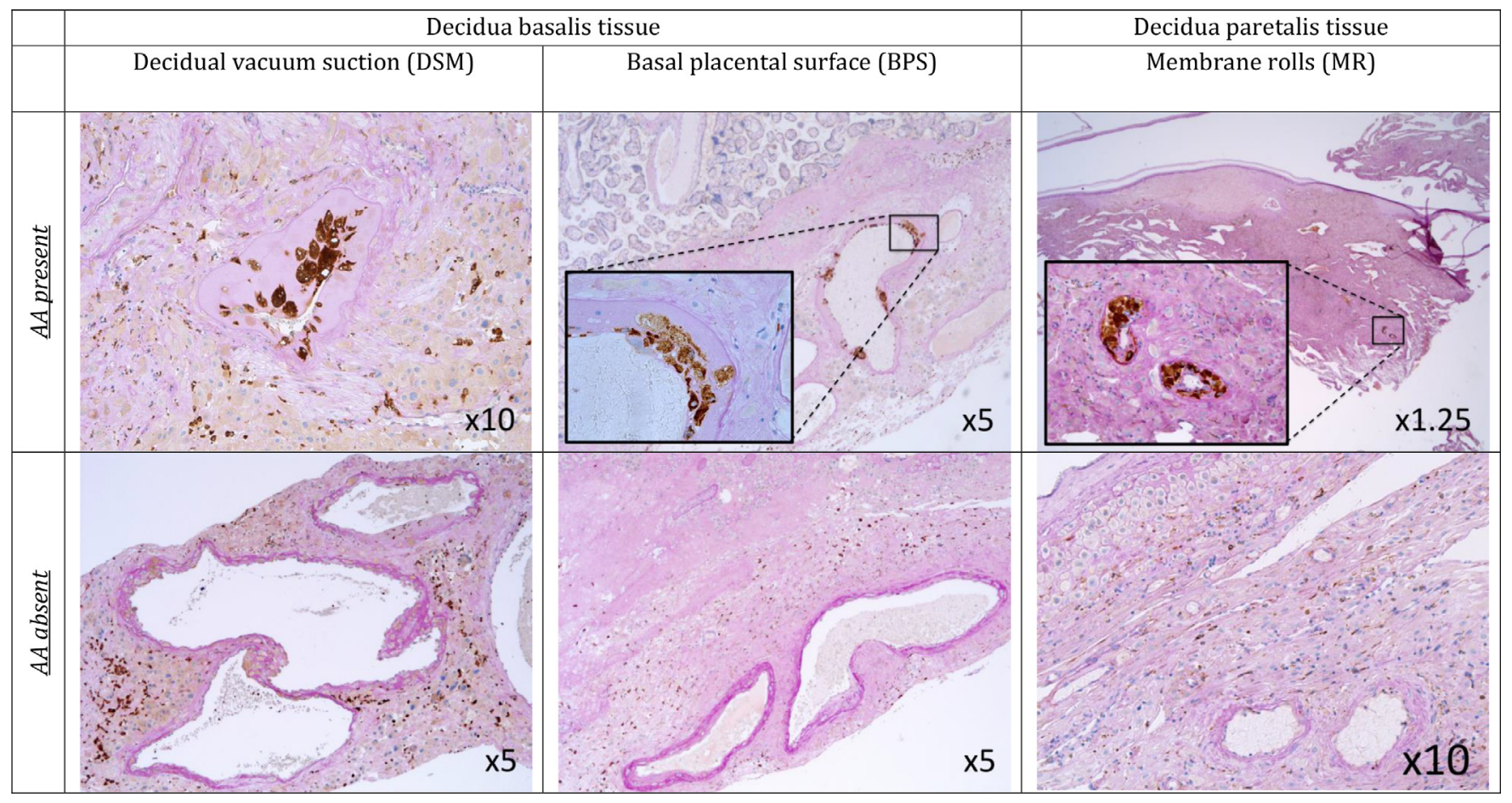

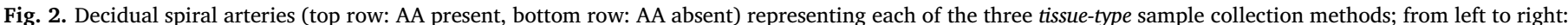

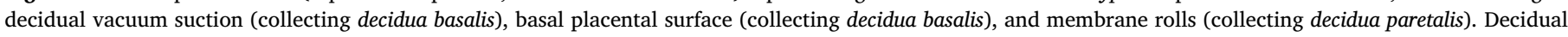

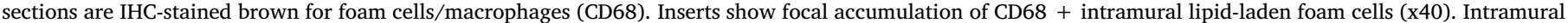
fibrinoid is identified using histochemical staining with Periodic acid Schiff (PAS).

methods for the 102 preeclamptic women where spiral arteries were found. Sampling from 37 women showed acute atherosis detected in the spiral artery wall(s) of at least one tissue sample type (Fig. 3). Among the $37 \mathrm{PE}$ women with AA, only one woman had AA lesions present in all three decidual samples types. All 8 women with AA presence in basal plate sections also had AA in their decidua basalis vacuum suction sample. However, the basal plate sections missed identifying 25 of the AA lesions present in the decidua vacuum suction collected samples.
Despite a satisfactory rate of decidua parietalis spiral artery detection in the placental fetal membrane roll (MR) samples (one or more arteries detected in $71 \%$ of the 107 samples), the AA rate in the MR was only11\% (Table 1). In four women, AA was detected in MR tissue alone (Fig. 3.). Taking membrane samples as a whole, $80 \%$ (317 out of 337) of arteries counted had diameters of $<100 \mu \mathrm{m}$, in keeping with the arteries not having undergone trophoblast remodeling (data not shown).

Most of MR arteries containing AA were $>100 \mu \mathrm{m}$ (in 7 of 8 
Table 1

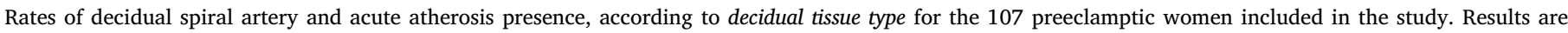

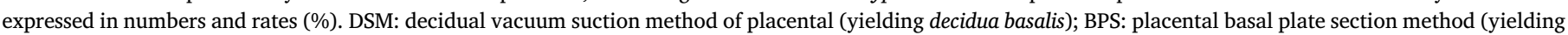
decidua basalis); MR: membrane roll (yielding decidua parietalis).

\begin{tabular}{|c|c|c|c|c|c|}
\hline \multirow{2}{*}{$\begin{array}{l}\text { Tissue } \\
\text { Sampling method }\end{array}$} & \multicolumn{2}{|l|}{ Decidua basalis } & \multirow{2}{*}{$\begin{array}{l}\text { Decidua parietalis } \\
\text { MR }\end{array}$} & \multicolumn{2}{|c|}{ Decidua basalis + parietalis } \\
\hline & DSM & BPS & & $\mathrm{MR}+\mathrm{BPS}$ & $\mathrm{DSM}+\mathrm{BPS}+\mathrm{MR}$ \\
\hline Spiral artery detection rate & $94 / 107(87 \%)$ & $53 / 107(50 \%)$ & 76/107 (71\%) & $85 / 107(79 \%)$ & $102 / 107(95 \%)$ \\
\hline Acute atherosis rate (of samples with spiral arteries present) & $33 / 94(35 \%)$ & $8 / 53(15 \%)$ & $8 / 76(11 \%)$ & $15 / 85(18 \%)$ & $37 / 102(36 \%)$ \\
\hline
\end{tabular}

Statistical significance of spiral artery detection rate differences; DSM vs BPS $\mathrm{p}<0.001$, DSM vs MR $\mathrm{p}=0.007$, BPS vs MR $\mathrm{p}=0.001$.

Statistical significance of AA detection differences; DSM vs BPS $\mathrm{p}=0.012$, DSM vs MR $\mathrm{p}<0.001$, BPS vs MR $\mathrm{p}=0.583$.

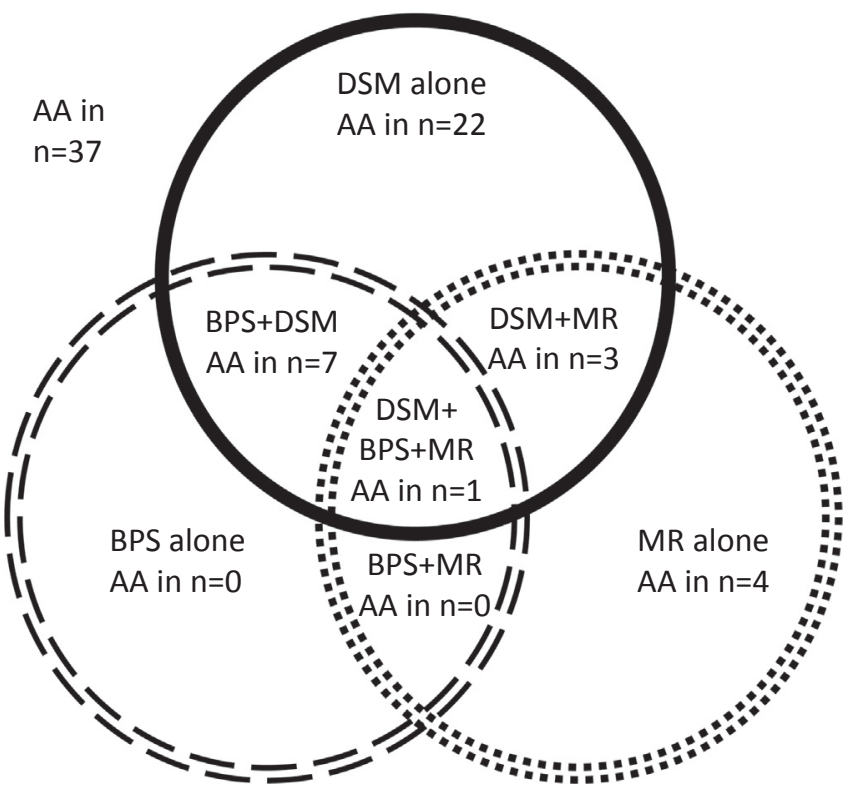

Fig. 3. Venn diagram representation of AA detection in uteroplacental tissue types for the 37 preeclamptic women with AA present. No AA was detected in any of the 3 decidual tissue types for the remaining 65 preeclamptic pregnancies with spiral arteries detected. DSM: Decidual vacuum suction method (collecting decidua basalis), BPS: Basal plate placenta section (collecting decidua basalis), MR: membrane rolls (collecting decidua parietalis).

women, and in 15 of 20 arteries counted containing AA), as opposed to only $17 \%$ (53 of 317) of MR arteries without AA, counted in the total of decidua parietalis samples (data not shown). Irrespective of AA, $69 \%$ (47 of 68) of MR artery sections with a diameter $>100 \mu \mathrm{m}$ were located proximal to the placental margin (within the first complete "turn" of the membrane roll, data not shown). None of the MR arterial walls contained endovascular trophoblasts.

\subsection{Association of $A A$ with clinical pregnancy parameters, according to tissue-type}

Table 2 presents the clinical parameters according to the detection of AA in one or more of the three tissue types evaluated. When combining samples from all three tissue types for each woman (DSM + BPS + MR), AA presence was significantly associated with a lower gestational age at delivery $(\mathrm{p}=0.007)$, and with a lower birthweight percentile ( $\mathrm{p}=0.034$ ), compared to women without AA (spiral arteries present) (Fig. 4.a). When evaluated separately, only presence of AA in DSM samples of decidua basalis associated significantly with lower gestational age at delivery and lower birthweight percentile ( $\mathrm{p}=0.025$ and $\mathrm{p}=0.039$ respectively, Fig. 4.b). When combining decidua basalis and parietalis tissue types collected in clinical histopathology routine (BPS + MR), presence of AA associated with a significantly lower gestational age at delivery (but not birthweight percentile), compared to women where AA was absent ( $p=0.025$, Fig. $4 \mathrm{c}$ ).

Rates of fetal growth restriction (FGR, defined as a birth weight percentile below $3 \%$ and/or antenatal pathological fetal Dopplers) were high in pregnancies with AA presence either in BPS (100\%) and MR (75\%), but not significantly higher compared to samples where AA was absent in either BPS or MR (or combination thereof). Only in DSM decidua basalis samples was FGR significantly associated with AA presence (AA present vs AA absent: $79 \%$ vs $40 \%$ FGR, $p=0.0004$, Table 2).

Pathological fetal Dopplers antenatally, regardless of birth weight percentile, was significantly more frequent in preeclamptic women with AA in combined BPS and MR as compared to lack of AA in these tissues ( $53 \%$ vs $23 \%$ FGR p $=0.0279$, Table 2 ), but not in DSM ( $39 \%$ vs $20 \%$ FGR, $\mathrm{p}=0.088$ ). In subanalyses, this decidual AA and pathological fetal Dopplers association was only valid for BPS decidua basalis AA ( $\mathrm{p}=0.0328$ ), not for MR decidua parietalis AA (data not shown).

We found no significant associations between AA presence (identified in either single or combinations of decidual tissue collection methods) and other clinical parameters associated with preeclampsia risk, such as maternal age, maternal BMI, mean blood pressure in the first half of pregnancy, infant sex, primigravidity, or previous history of PE.

\section{Discussion}

Our study shows that vacuum suctioned decidua basalis tissue has highest detection rate of AA (37\%), when compared to the two other, more conventional, spiral artery sampling methods (basal plate placenta sections and membrane roll sections). This is the first study where these specified sample collection methods - applied to each woman delivered - are compared for the detection rate of decidual AA.

The homogeneity and completeness of the sample tissue types is the advantage of this study, as is the predefined definition of AA in the tissues evaluated, with previously demonstrated satisfactory inter- and intra-observer agreement for AA detection in decidua basalis spiral arteries [6]. Our findings, representing a relatively large preeclampsia group from one single hospital, confirm our previous conclusion, based on comparing different groups of women: the decidua vacuum suction method is the superior method for the detection of decidual spiral arteries [7] compared to conventional pathology sections from delivered placentas.

This study's findings are in line with our previous spiral artery detection work, demonstrating DSM as superior to BPS and placenta bed biopsies [7], the latter not investigated in the present study. Placenta bed biopsies would have enabled assessment also of myometrial tissue, not available in our decidual suction specimens. However, AA rates have previously been shown to be higher in the decidual, compared to the deeper myometrial, portion of the placental bed [24,25]. Our observations of AA detected in both small and larger caliber spiral arteries in MR confirms previous observations $[3,5,26]$ indicating that AA development is not dependent upon trophoblast-mediated spiral artery remodeling. This supports our concept that different mechanisms may 


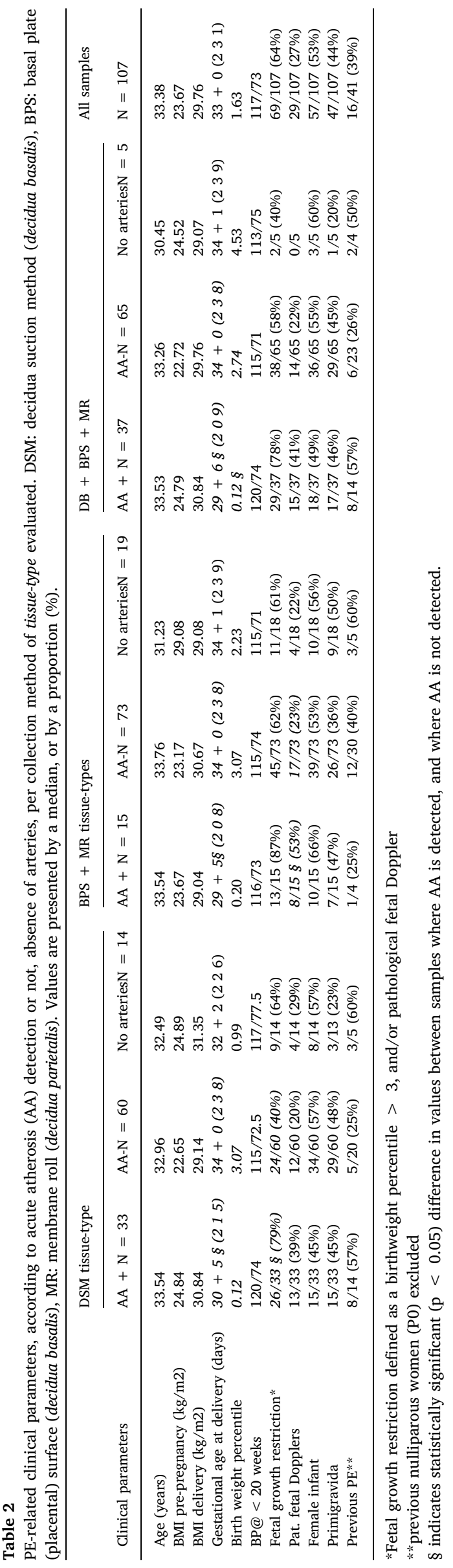

lead to AA development [27]. The AA lesions tend to be closer to the placenta in the membrane rolls, and the decidual spiral arteries seem to have higher burden of AA than myometrial parts. We suggest that arterial proximity to extravillous trophoblasts during placentation may be one of the mechanisms that trigger the development of the AA lesion.

Khong's use of an alternative "en face" BPS biopsies (meaning sectioning of decidua basalis parallel to the maternal placenta surface) demonstrated an increase in the detection rate of spiral arteries compared to investigations of conventional full thickness of placental parenchyma, including basal plate decidual biopsies. Presumably this is because the en face biopsies provided a greater surface area of decidua basalis for evaluation [28]. In our study, we used only one full thickness placental section for examination of the maternal plate (BPS), comparing this to one section from the decidua tissue collected by vacuum suction (DSM). AA lesions are focal, and do not affect spiral arteries universally [22], and its presence in the placental bed is associated with histopathological areas of placental vascular underperfusion and infarcts [29]. Though not consistently, the surface area of decidua contained on the slide was in our study larger for DSM compared to BPS. It is possible that increasing the number of BPS sections evaluated per woman participant (increasing the decidual surface area evaluated) would imply a concurrent rise in AA detection. Increased decidua basalis AA detection was not commented upon by Khong, despite his demonstration of increased spiral artery detection [28]. In our study, we found similar number of spiral arteries in slides where AA was detected when comparing DSM to BPS tissue sections (data not shown). This implies that the lower detection rate of AA in BPS as compared to DSM is likely not only explained by a difference in number of spiral arteries present per sample.

No cases of AA detection was found exclusive to the decidual basal plate section alone, but was always paralleled with AA presence in DSM biopsies. The basal plate placental section reflects the same placental bed where the remaining decidual tissue was collected by vacuum suction after placenta removal. The lower detection rate for AA in routine sampling alone (BPS and MR) may explain the disparity between lower AA detection rate reported from research groups examining solely BPS and MR [5], and the higher AA rates found in our decidua basalis vacuum suction studies $[5,6]$. A drawback with the decidual vacuum suction method as the optimal source for AA diagnosis is however the need for delivery by caesarean section, which should only be undertaken if indicated for a maternal and fetal health benefit.

Our current study's AA detection rate of $18 \%$ in combined BPS and MR samples is comparable to the detection rate of $10 \%$ reported by Kim et al in the hitherto largest published study of AA detection using H\&E stained BPS and MR tissues. Kim et al suggest in their work a potential under-reporting of AA due to absence of confirmatory staining for foam cells, namely immunostaining decidual sections with CD68, alternatively oil-red $\mathrm{O}$ special staining of frozen samples [5].

Our study showed a significant association between AA presence and three feto-placental clinical outcomes that are common in earlyonset and clinically severe preeclampsia: low gestational age at delivery, low birthweight percentile, and pre-delivery pathological fetal Dopplers. Low birthweight percentile and pathological Dopplers are also features of fetal growth restriction and of placental dysfunction. Women with a history of severe preeclampsia, especially when complicated by preterm delivery and/or fetal growth restriction, represent the group with highest epidemiological risk for premature cardiovascular mortality, up to 8-fold [10]. Another study of decidual AA found a significant association to maternal vascular underperfusion [30], which is in line with our conclusion of decidual AA being associated with clinical placental dysfunction characteristics. Our subanalysis showing no association between pathological fetal Dopplers and AA presence in MR decidual tissue alone, is consistent with the findings of Khong et al. [31], where AA was not found in MR decidual samples in pregnancies with growth restricted babies [31].

Our group has recently shown that decidual AA is significantly 


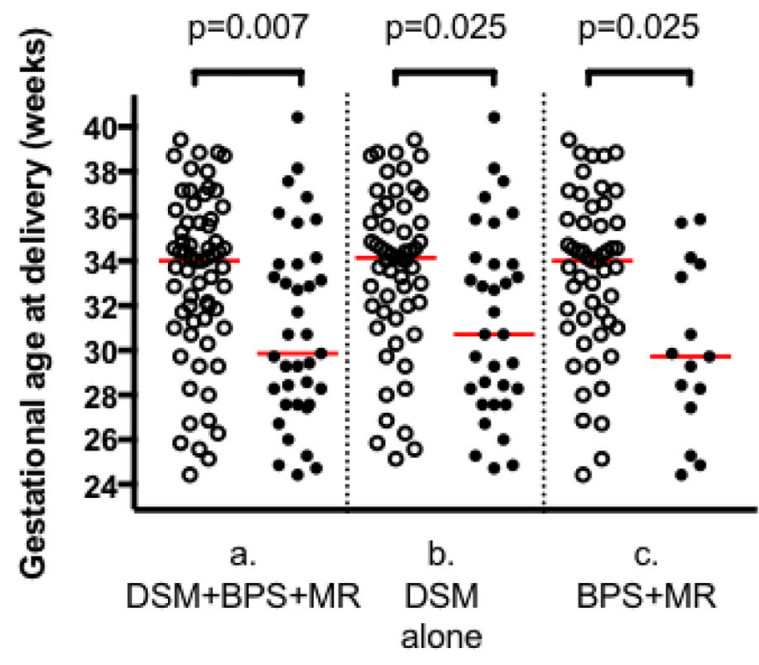

- yes arteries, no AA

- yes arteries, yes AA

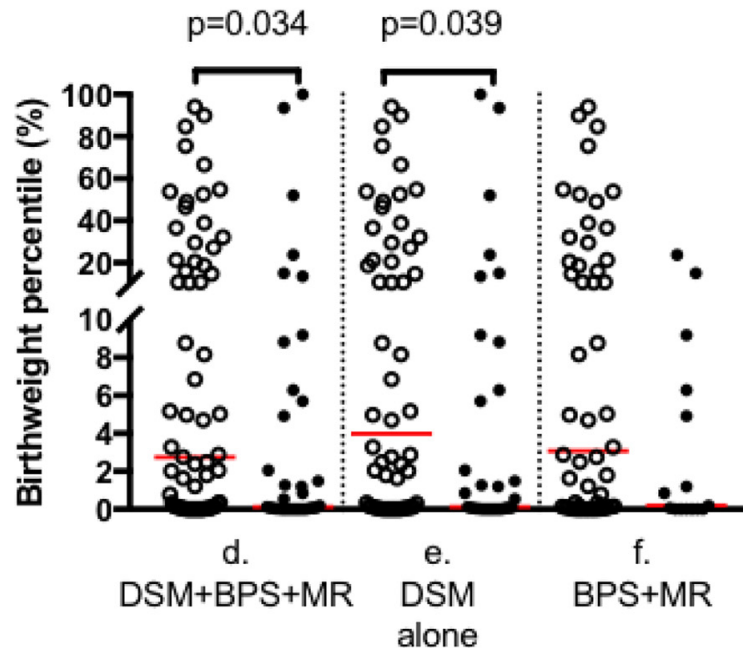

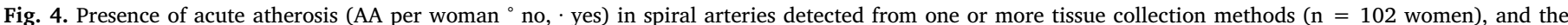

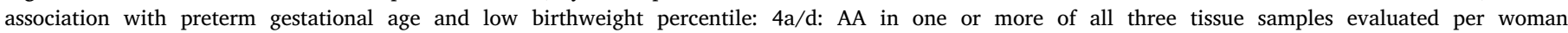

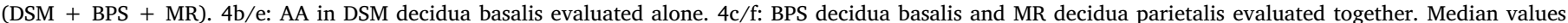
shown in horizontal bar. $\mathrm{p}$ values shown when $\mathrm{p}<0.05$. DSM: decidual vacuum suction method. BPS: placental basal plate section. MR: membrane roll.

associated with the combination of maternal KIR-B haplotype and fetal HLA-C2 [32]. This is suggestive of an impact of alloimmune interactions at the decidual materno-fetal interface, as another mechanism promoting decidual AA vascular lesions and affecting placental function. In general, there is an intimate relationship between the immune and the cardiovascular system, both during [33] and outside pregnancy $[34,35]$. We have suggested that AA may serve as a positive screening test for parous women with increased risk for premature CVD $[12,27,36,37]$. This is a new concept being tested by ourselves, also gaining interest among other investigators [38]. The American Heart Association has since 2011 included a history of pregnancy hypertension as a risk factor for cardiovascular disease (CVD) [39]. Our future aim is to investigate whether decidual acute atherosis could serve as a personalized targeting of young women at highest future cardiovascular risk, facilitating intensive personalized prevention and follow-up programs.

\section{Conclusion}

Compared to conventional sampling, vacuum suctioned decidua basalis tissue, obtained at cesarean section, remains the most reliable method for the identification of decidual spiral arteries and subsequent detection of spiral artery acute atherosis. Compared to preeclamptics without identified AA lesions, AA presence in the spiral arteries of decidua basalis was associated with parameters of impaired placental function. Whether presence and quantity of AA in decidua basalis and/ or parietalis identifies preeclamptic women with high risk of longterm atherosclerotic CVD, remains to be seen. Our study shows, however, that the vacuum suction decidual basalis material is especially well suited in studies trying to solve this question.

\section{Declaration of Competing Interest}

The authors declare that they have no known competing financial interests or personal relationships that could have appeared to influence the work reported in this paper. 


\section{Acknowledgements}

We would like to thank administrator for the Oslo Pregnancy Biobank, Lise Levy, for assisting with sample procurement and biobank handling. We would also like to thank Linn Buer and Giang Nguyen, Department of Pathology, Oslo University Hospital, for the sectioning and immunostaining of decidual samples. A special thanks to Professor Christopher WG Redman (Oxford, UK) for his guidance in the planning of this study. Lastly, thanks to the support of Lien My Diep, Biostatistician/Statistician, Oslo Centre for Biostatistics and Epidemiology, Oslo University Hospital, Oslo, Norway.

The Research Council of Norway funded part of this work (the PATH study: Pregnancy acute atherosis and future cardiovascular disease, grant ref. 230652) as did South-Eastern Norway Regional Health Authority (the HAPPY PATH study, grant ref. 2014026). Oslo University Hospital provided PhD salary (PAK) and further research support.

\section{Appendix A. Supplementary data}

Supplementary data to this article can be found online at https:// doi.org/10.1016/j.preghy.2019.11.007.

\section{References}

[1] A.T. Hertig, Vacular pathology in hypertensive albuminuric toxemias of pregnancy, Clinics 4 (1945) 602-614.

[2] F. De Wolf, W.B. Robertson, I. Brosens, The ultrastructure of acute atherosis in hypertensive pregnancy, Am. J. Obstet. Gynecol. 123 (2) (1975) 164-174.

[3] P.M. Zeek, N.S. Assali, Vascular changes in the decidua associated with eclamptogenic toxemia of pregnancy, Am. J. Clin. Pathol. 20 (12) (1950) 1099-1109.

[4] D.U. Stevens, S. Al-Nasiry, J. Bulten, M.E. Spaanderman, Decidual vasculopathy and adverse perinatal outcome in preeclamptic pregnancy, Placenta 33 (8) (2012) 630-633.

[5] Y.M. Kim, P. Chaemsaithong, R. Romero, M. Shaman, C.J. Kim, J.S. Kim, F. Qureshi, S.M. Jacques, A.I. Ahmed, et al., The frequency of acute atherosis in normal pregnancy and preterm labor, preeclampsia, small-for-gestational age, fetal death and midtrimester spontaneous abortion, J. Matern Fetal. Neonatal Med. 28 (17) (2015) 2001-2009.

[6] P. Alnaes-Katjavivi, F. Lyall, B. Roald, C.W. Redman, A.C. Staff, Acute atherosis in vacuum suction biopsies of decidua basalis: an evidence based research definition, Placenta 37 (2016) 26-33.

[7] N.K. Harsem, A.C. Staff, L. He, B. Roald, The decidual suction method: a new way of collecting decidual tissue for functional and morphological studies, Acta Obstet. Gynecol. Scand. 83 (8) (2004) 724-730.

[8] L. Bellamy, J.P. Casas, A.D. Hingorani, D.J. Williams, Pre-eclampsia and risk of cardiovascular disease and cancer in later life: systematic review and meta-analysis, BMJ 335 (7627) (2007) 974.

[9] J.A. Lykke, J. Langhoff-Roos, B.M. Sibai, E.F. Funai, E.W. Triche, M.J. Paidas, Hypertensive pregnancy disorders and subsequent cardiovascular morbidity and type 2 diabetes mellitus in the mother, Hypertension 53 (6) (2009) 944-951.

[10] A.C. Staff, C.W. Redman, D. Williams, P. Leeson, K. Moe, B. Thilaganathan, P. Magnus, E.A. Steegers, E.Z. Tsigas, et al., Pregnancy and long-term maternal cardiovascular health: progress through harmonization of research cohorts and biobanks, Hypertension 67 (2) (2016) 251-260.

[11] H.C. Stary, A.B. Chandler, S. Glagov, J.R. Guyton, W. Insull Jr., M.E. Rosenfeld, S.A. Schaffer, C.J. Schwartz, W.D. Wagner, et al., A definition of initial, fatty streak, and intermediate lesions of atherosclerosis. A report from the Committee on Vascular Lesions of the Council on Arteriosclerosis, Am. Heart Assoc. Circ. 89 (5) (1994) 2462-2478.

[12] A.C. Staff, C.W. Redman, IFPA Award in placentology lecture: preeclampsia, the decidual battleground and future maternal cardiovascular disease, Placenta 35 (Suppl) (2014) S26-S31.

[13] J.M. Roberts, G. Pearson, J. Cutler, M. Lindheimer, Summary of the NHLBI working group on research on hypertension during pregnancy, Hypertension 41 (3) (2003)
$437-445$

[14] S.L. Johnsen, S. Rasmussen, T. Wilsgaard, R. Sollien, T. Kiserud, Longitudinal reference ranges for estimated fetal weight, Acta Obstet. Gynecol. Scand. 85 (3) (2006) 286-297.

[15] D. Arduini, G. Rizzo, Normal values of pulsatility index from fetal vessels: a cross sectional study on 1556 healthy fetuses, J. Perinat. Med. 18 (3) (1990) 165-172.

[16] G.R. DeVore, The importance of the cerebroplacental ratio in the evaluation of fetal well-being in SGA and AGA fetuses, Am. J. Obstet. Gynecol. 213 (1) (2015) 5-15.

[17] A.C. Staff, T. Ranheim, J. Khoury, T. Henriksen, Increased contents of phospholipids, cholesterol, and lipid peroxides in decidua basalis in women with preeclampsia, Am. J. Obstet. Gynecol. 180 (3 Pt 1) (1999) 587-592.

[18] G. Turowski, L.N. Berge, L.B. Helgadottir, E.M. Jacobsen, B. Roald, A new, clinically oriented, unifying and simple placental classification system, Placenta 33 (12) (2012) 1026-1035

[19] J.F. McManus, Histological and histochemical uses of periodic acid, Stain Technol 23 (3) (1948) 99-108.

[20] R. Pijnenborg, E. Ball, J.N. Bulmer, M. Hanssens, S.C. Robson, L. Vercruysse, In vivo analysis of trophoblast cell invasion in the human, Methods Mol. Med. 122 (2006) $11-44$.

[21] Ramsey EM DM. Placental vasculature and circulation. Anatomy, physiology, radiology, clinical aspects atlas textbook. Stuttgart: Georg Thieme Publishers; 1980. p. 20, p.13.

[22] R. Pijnenborg, L. Vercruysse, M. Hanssens, The uterine spiral arteries in human pregnancy: facts and controversies, Placenta 27 (9-10) (2006) 939-958.

[24] J.W. Meekins, R. Pijnenborg, M. Hanssens, I.R. McFadyen, A. van Asshe, A study of placental bed spiral arteries and trophoblast invasion in normal and severe preeclamptic pregnancies, Br. J. Obstet. Gynaecol. 101 (8) (1994) 669-674.

[25] B.L. Sheppard, J. Bonnar, The ultrastructure of the arterial supply of the human placenta in pregnancy complicated by fetal growth retardation, Br. J. Obstet. Gynaecol. 83 (12) (1976) 948-959.

[26] M. Maqueo, J. Chavezazuela, M. Dosaldelavega, Placental pathology in eclampsia and preeclampsia, Obstet. Gynecol. 24 (1964) 350-356.

[27] A.C. Staff, G.M. Johnsen, R. Dechend, C.W. Redman, Preeclampsia and uteroplacental acute atherosis: immune and inflammatory factors, J. Reprod. Immunol. 101-102 (2014) 120-126.

[28] T.Y. Khong, H.M. Chambers, Alternative method of sampling placentas for the assessment of uteroplacental vasculature, J. Clin. Pathol, 45 (10) (1992) 925-927.

[29] I. Brosens, M. Renaer, On the pathogenesis of placental infarcts in pre-eclampsia, J. Obstet. Gynaecol. Br. Commonw. 79 (9) (1972) 794-799.

[30] Y.M. Kim, P. Chaemsaithong, R. Romero, M. Shaman, C.J. Kim, J.S. Kim, F. Qureshi, S.M. Jacques, A.I. Ahmed, et al., Placental lesions associated with acute atherosis, J. Matern Fetal Neonatal Med. 1-29 (2014).

[31] T.Y. Khong, Acute atherosis in pregnancies complicated by hypertension, small-forgestational-age infants, and diabetes mellitus, Arch. Pathol. Lab. Med. 115 (7) (1991) 722-725.

[32] G.M. Johnsen, G.L. Storvold, J.J.M. Drabbels, G.W. Haasnoot, M. Eikmans, M.J. Spruyt-Gerritse, P. Alnaes-Katjavivi, S.A. Scherjon, C.W.G. Redman, et al., The combination of maternal KIR-B and fetal HLA-C2 is associated with decidua basalis acute atherosis in pregnancies with preeclampsia, J. Reprod. Immunol. 129 (2018) 23-29.

[33] A.S. Care, S.L. Bourque, J.S. Morton, E.P. Hjartarson, S.A. Robertson, S.T. Davidge, Reduction in regulatory $\mathrm{T}$ cells in early pregnancy causes uterine artery dysfunction in mice, Hypertension 72 (1) (2018) 177-187.

[34] N. Sasaki, T. Yamashita, M. Takeda, K. Hirata, Regulatory T cells in atherogenesis, J. Atheroscler. Thromb. 19 (6) (2012) 503-515.

[35] D.A. Chistiakov, A.N. Orekhov, Y.V. Bobryshev, Immune-inflammatory responses in atherosclerosis: role of an adaptive immunity mainly driven by T and B cells, Immunobiology 221 (9) (2016) 1014-1033.

[36] A.C. Staff, R. Dechend, R. Pijnenborg, Learning from the placenta: acute atherosis and vascular remodeling in preeclampsia-novel aspects for atherosclerosis and future cardiovascular health, Hypertension 56 (6) (2010) 1026-1034.

[37] A.C. Staff, R. Dechend, C.W. Redman, Review: Preeclampsia, acute atherosis of the spiral arteries and future cardiovascular disease: two new hypotheses, Placenta 34 (Suppl) (2013) S73-S78.

[38] D.U. Stevens, S. Al-Nasiry, M.M. Fajta, J. Bulten, A.P. van Dijk, M.J. van der Vlugt, W.J. Oyen, J.M. van Vugt, M.E. Spaanderman, Cardiovascular and thrombogenic risk of decidual vasculopathy in preeclampsia, Am. J. Obstet. Gynecol. 210 (6) (2014) 545-546.

[39] L. Mosca, E J. Benjamin, K. Berra, J.L. Bezanson, R.J. Dolor, D.M. Lloyd-Jones, L.K. Newby, I.L. Pina, V.L. Roger, et al., Effectiveness-based guidelines for the prevention of cardiovascular disease in women-2011 update: a guideline from the American Heart Association, J. Am. Coll. Cardiol. 57 (12) (2011) 1404-1423. 